\title{
Progressive damage on high resolution computed tomography despite stable lung function in cystic fibrosis
}

\author{
P.A. de Jong*, Y. Nakano", M.H. Lequin ${ }^{\Uparrow}$, J.R Mayo ${ }^{+}$, R. Woods ${ }^{\#}$, P.D. Paré\#, \\ H.A.W.M. Tiddens*
}

Progressive damage on high resolution computed tomography despite stable lung function in cystic fibrosis. P.A. de Jong, Y. Nakano, M.H. Lequin, J.R Mayo, R. Woods, P.D. Paré, H.A.W.M. Tiddens. (C) ERS Journals Ltd 2004.

ABSTRACT: For effective clinical management of cystic fibrosis (CF) lung disease it is important to closely monitor the start and progression of lung damage. The aim of this study was to investigate the ability of high-resolution computed tomography (HRCT) scoring systems and pulmonary function tests (PFT) to detect changes in lung disease.

CF children $(n=48)$ had two HRCT scans in combination with two PFT 2 yrs apart. Their scans were scored using five scoring systems (Castile, Brody, Helbich, Santamaria and Bhalla). "Sensitivity" was defined as the ability to detect disease progression.

In this group of children, HRCT scores worsened. PFT remained unchanged or improved. Of the HRCT parameters, mucous plugging and the severity, extent and peripheral extension of bronchiectasis worsened significantly. Relationships between changes in HRCT scores and PFT were weak. Substantial structural lung damage was evident in some children who had normal lung function.

These data show that high-resolution computed tomography is more sensitive than pulmonary function tests in the detection of early and progressive lung disease, and suggest that high-resolution computed tomography may be useful in the follow up of cystic fibrosis children and as an outcome measure in studies that aim to reduce lung damage.

Eur Respir J 2004; 23: 93-97.
*Dept of Paediatric Pulmonology and "Dept of Paediatric Radiology, Sophia Children's Hospital, Erasmus Medical Centre Rotterdam, Rotterdam, The Netherlands. " UBC McDonald Research Laboratories and iCAPTURE Centre, St Paul's Hospital, and ${ }^{+}$Dept of Radiology, Vancouver Hospital and Health Science Center, Vancouver, BC, Canada.

Correspondence: H.A.W.M. Tiddens, Sophia Children's Hospital, Dept of Pediatric Pulmonology, Dr Molewaterplein 603015 GJ Rotterdam, The Netherlands.

Fax: 31 104636801

E-mail: h.tiddens@erasmusmc.nl

Keywords: Bronchiectasis, children, cystic fibrosis, high-resolution computed tomography, lung structure, pulmonary function test

Received: January 172003

Accepted after revision: August 282003

This work was supported in part by a grant from the NHL BI (HL64068-03). P.A. de Jong was supported by a Gerrit Jan Mulder Stichting Fellowship (GJM-stichting, Erasmus Medical Centre, Rotterdam, The Netherlands).
In cystic fibrosis (CF), chronic bacterial infection leads to progressive structural lung damage and to pulmonary dysfunction. It is generally accepted that early and aggressive therapy could delay the progression of lung disease. To evaluate the efficacy of such treatment it is important to monitor the progression of lung damage closely. Pulmonary function tests (PFT) are considered the gold standard for the monitoring of lung disease in children of aged $\geqslant 6 \mathrm{yrs}$. However, since lung function is only indirectly related to lung structure, it is likely that high-resolution computed tomography (HRCT) is more sensitive than PFT in the detection of structural changes compared with PFT [1]. Some CF centres have adapted periodic HRCT on a routine basis to evaluate the progression of $\mathrm{CF}$ lung disease in combination with PFT. However, it is unclear what method should be used to analyse the HRCT and whether HRCT is more sensitive in detecting pulmonary disease progression in CF. It could be that PFT and HRCT provide complementary information and should be performed in parallel to assess progression. The aim of this study was to investigate the sensitivity of five different HRCT-scoring systems and PFT to detect changes in $\mathrm{CF}$ structural lung disease over time.
Methods

\section{Study population}

Since 1996, Sophia Children's Hospital (Rotterdam, The Netherlands), a tertiary academic hospital has monitored CF patients using annual PFT and biennial HRCT scans. All children with $\mathrm{CF}$ who had two HRCT scans (HRCT1 and HRCT2) in combination with two routine PFT (PFT1 and PFT2) were selected for this follow-up study $(n=48)$. CF was diagnosed by a positive sweat test and/or genotyping for known CF mutations and/or an abnormal potential difference measured across the nasal mucosa. The ethical review board of the hospital approved the study.

\section{Lung structure}

All HRCT scans were acquired using a GE Prospeed SX scanner (General Electric Medical Systems, Milwaukee, WI, USA). During the scanning procedure, children were not sedated, they were scanned in the supine position, and 
instructed to take a deep breath and hold it for $\geqslant 5 \mathrm{~s}$. A complete HRCT series contained $\sim 251-\mathrm{mm}$ thick slices at 10 -mm intervals from lung apex to lung base. Scanning parameters were $120 \mathrm{kV}, 160 \mathrm{~mA}$ ( $<9$ yrs of age $120 \mathrm{~mA}$ ), 1-s scanning time and a field of view of $350 \mathrm{~mm}(<9$ yrs of age $250 \mathrm{~mm})$. Scans were reconstructed with a detail reconstruction algorithm (General Electric Medical Systems) and printed using window width -600 Hounsfield units (HU) and window level 1,500 HU. All HRCT scans were scored in random order by an experienced single observer blinded as to the date of the scan, patient identification and the PFT. Five different HRCT-scoring systems were used: CASTILE et al. [2], BRODY et al. [3], Helbich et al. [4], SANTAMARIA et al. [5], and BHALlA et al. [6]. In a previous study, in which the observer of this study participated, these five scoring systems were shown to be reliable between and within observers [7]. All slices were used to score the lung lobes and the lingula $[2,3]$ or the bronchopulmonary segments of the lung [4-6]. The systems score bronchiectasis, bronchial wall thickening, mucous plugging, atelectasis, bulla, cysts, consolidation, acinar nodules, septal thickening and/or air trapping in a semiquantitative fashion. A score of zero means "no abnormalities" in each system. Maximal scores are 92, 100, 27, 29 and 25 for Castile, Brody, Helbich, Santamaria and Bhalla scores, respectively.

\section{Lung function}

All PFT were done within 1 month of the HRCT scanning using a Jäger diagnostic system (MasterLab, Jäger, Germany). Most scans were performed on the same day (71 of 96 scans) as the PFT. All HRCT scans were done as part of a routine check-up and thus patients were scanned in a relatively stable condition and not during an exacerbation. PFT results were expressed as percentage of predicted values: forced expiratory volume in one second (FEV1) [8], forced vital capacity (FVC) [8], forced expiratory flow between $25 \%$ and $75 \%$ of expiratory vital capacity (FEF25-75\%) [9], airway resistance (Raw) [10], residual volume (RV) [10], total lung capacity (TLC) and RV/TLC [10]. The ratio FEV1/FVC and the ratio RV/TLC were expressed as a percentage (FEV1/FVC \% and RV/TLC $\%$ ). Twelve patients were younger than patients studied by WANG et al. [9] in the development of the prediction equation for FEF25-75\%. Body plethysmography (TLC, RV and Raw) was performed in 33 of the 48 children. Normal lung function was defined as a FEV1 of $>85 \%$ predicted.

\section{Lung structure and lung function over time}

Data obtained at the first evaluation are reported as HRCT1 and PFT1, and data obtained at the second evaluation are reported as HRCT2 and PFT2. $\triangle$ HRCT is the annual change for a scoring system ((HRCT2-HRCT1)/ time interval). To compare the annual changes of the five HRCT-scoring systems, $\triangle$ HRCT was also expressed as a percentage of the maximal obtainable HRCT score for that system. $\triangle \mathrm{PFT}$ is the annual change expressed as $\%$ pred for a lung function parameter ((PFT2-PFT1)/time interval). A positive value for $\triangle \mathrm{HRCT}$ indicates an increase of structural abnormalities and for $\triangle \mathrm{PFT}$ an improvement in lung function. $\triangle \mathrm{HRCT}$ and $\triangle \mathrm{PFT}$ were evaluated for the whole group and separately for children younger than and older than $10 \mathrm{yrs}$ of age. $\triangle \mathrm{HRCT}$ and $\triangle \mathrm{PFT}$ were also evaluated as a function of age and baseline disease severity (HRCT1 and FEV1\% pred).

\section{Statistical analysis}

For the purpose of this article, the term sensitivity is not used in the statistical sense but rather as a measure of the techniques' ability to track pulmonary disease progression in CF. It was assumed that, on average, CF lung disease would be progressive over 2 yrs and that the method that detected the largest change was most "sensitive". The relationships between HRCT1 and PFT1, HRCT2 and PFT2, between $\triangle \mathrm{HRCT}$ and $\triangle \mathrm{PFT}$ and between $\triangle \mathrm{HRCT}, \triangle \mathrm{PFT}, \mathrm{HRCT} 1$, PFT1 and age were evaluated using the Spearman correlation coefficient. t-tests (unpaired) were performed for $\triangle \mathrm{HRCT}$ and $\triangle$ PFT to determine whether HRCT and/or PFT changed significantly in the whole group and for children below and above 10 yrs of age. Wilcoxon-signed ranks tests were used to determine which of the HRCT abnormalities changed significantly. Statistical significance was set at a p-value of $<0.05$. Data are presented as mean \pm SD and range.

\section{Results}

\section{Study population}

Characteristics of the 48 children ( 28 male) are shown in table 1. At HRCT1, 15 children had normal lung function (31\%). HRCT scores were 13.7 $\pm 8.7,8.1 \pm 6.8,6.1 \pm 2.9$, $6.4 \pm 3.2$ and $6.1 \pm 2.9$ for Castile, Brody, Helbich, Santamaria and Bhalla, respectively.

\section{Lung structure and lung function over time}

All HRCT scores worsened significantly. The mean changes for the whole group expressed as a percentage of the maximal scores were 2.6, 2.2, 3.3, 3.1 and $3.5 \%$ per year for Castile, Brody, Helbich, Santamaria, and Bhalla, respectively. Spirometric parameters $(\triangle \mathrm{FEV} 1, \Delta \mathrm{FVC}, \triangle \mathrm{FEF} 25-75 \%$ and $\triangle \mathrm{FEV} 1 /$ FVC) did not change significantly. RV \% pred, RV/TLC $\%$ and RV/TLC \% pred decreased by $8.9 \%$ ( $\mathrm{p}=0.004), 2.4 \%$ $(p=0.0005)$ and $0.66 \%(p<0.0001)$ per year, respectively. Raw and TLC did not change significantly. The subgroup analysis of children below and above $10 \mathrm{yrs}$ of age showed the same results, with the exception that $\mathrm{RV} \%$ pred remained unchanged in the children $>10 \mathrm{yrs}$ of age. $\triangle \mathrm{HRCT}$ and $\triangle$ PFT did not differ as a function of baseline disease severity or age, with the exception of significant relationships between age and $\Delta \mathrm{RV} \%$ pred $(\mathrm{R}=0.53, \mathrm{p}=0.001)$ and between age and $\Delta \mathrm{RV} / \mathrm{TLC} \%(\mathrm{R}=0.46, \mathrm{p}=0.008)$.

Of the HRCT scan parameters only mucous plugging $(\mathrm{p}=0.001)$ and the severity $(\mathrm{p}=0.005)$, extent $(\mathrm{p}<0.0001)$ and peripheral extension $(\mathrm{p}=0.02)$ of bronchiectasis worsened significantly. Of the 48 patients, the severity of bronchiectasis increased in 15, the score for the extent of bronchiectasis increased in 19 and the score for the peripheral extension of bronchiectasis increased in eight. In only three patients there was a reduction of the severity of bronchiectasis (in all three cases, the airway lumen diameter changed from $\sim 2-3$-times vessel diameter to $\sim 1-2$-times vessel diameter). One patient showed a reduction in the peripheral extension score.

\section{Correlation between lung structure and lung function}

Cross-sectional data showed a significant correlation between HRCT1 and first FEV1 $(\mathrm{R}<-0.49, \mathrm{p}<0.0001)$, and between HRCT2 and second FEV1 $(\mathrm{R}<-0.58, \mathrm{p}<0.0001)$. Longitudinal data showed significant but weak correlation 


\begin{tabular}{lccc}
\hline & First HRCT & Second HRCT & Mean annual change \\
\hline Age yrs & $11.05 \pm 3.30$ & $13.04 \pm 3.30$ & Interval 1.99 \\
Body height m & $1.37 \pm 0.21$ & $1.47 \pm 0.20$ & $0.047^{*}$ \\
Body weight kg & $32.5 \pm 12.1$ & $38.6 \pm 13.9$ & $3.46^{*}$ \\
FEV1\% pred & $74.3 \pm 18.2$ & $76.0 \pm 21.0$ & 1.24 \\
FVC \% pred & $85.5 \pm 15.7$ & $85.1 \pm 16.2$ & -0.11 \\
FEF25-75\% pred & $58.8 \pm 31.2$ & $59.9 \pm 32.0$ & -1.30 \\
FEV1/FVC \% & $75.4 \pm 10.6$ & $77.6 \pm 9.8$ & 0.39 \\
Raw \% pred & $136.1 \pm 61.8$ & $143.4 \pm 76.0$ & 0.10 \\
RV\% pred & $136.6 \pm 42.1$ & $129.9 \pm 40.2$ & $-8.49^{*}$ \\
TLC \% pred & $98.0 \pm 13.2$ & $96.8 \pm 11.2$ & -1.06 \\
RV/TLC \% & $33.2 \pm 9.4$ & $31.6 \pm 9.7$ & $-2.11^{*}$ \\
Castile score [2] & $17.7 \pm 9.7$ & $22.4 \pm 11.4$ & $2.39^{*}$ \\
Brody score [3] & $12.1 \pm 8.7$ & $16.3 \pm 10.2$ & $2.21^{*}$ \\
Helbich score [4] & $8.4 \pm 3.7$ & $10.1 \pm 3.8$ & $0.88^{*}$ \\
Santamaria score [5] & $8.9 \pm 4.0$ & $10.7 \pm 4.1$ & $0.91^{*}$ \\
Bhalla score [6] & $8.2 \pm 3.4$ & $10.0 \pm 3.6$ & $0.87^{*}$ \\
\hline
\end{tabular}

Data are presented as mean \pm SD. HRCT: high-resolution computed tomography; FEV1: forced expiratory volume in one second; \% pred: $\%$ predicted; FVC: forced vital capacity; FEF25-75\%: forced mid-expiratory flow; Raw: airway resistance; RV: residual volume; TLC: total lung capacity. *: p<0.05 in $1 \mathrm{yr}$ with the one-sample t-test (unpaired).

between $\triangle \mathrm{PFT}$ and $\triangle \mathrm{HRCT}: \triangle \mathrm{FEV} 1 / \mathrm{FVC}$ versus $\triangle$ Santamaria $(\mathrm{R}=-0.31, \mathrm{p}=0.04), \Delta \mathrm{R}$ aw versus $\Delta$ Helbich $(\mathrm{R}=0.37, \mathrm{p}=0.04)$, $\Delta \mathrm{R}$ aw versus $\Delta$ Santamaria $(\mathrm{R}=0.38, \mathrm{p}=0.03), \Delta \mathrm{RV}$ versus $\Delta$ Brody $(\mathrm{R}=0.35, \mathrm{p}=0.04), \Delta \mathrm{RV} / \mathrm{TLC}$ versus $\Delta \mathrm{Brody}(\mathrm{R}=0.38$, $\mathrm{p}=0.03)$, and $\Delta \mathrm{RV} / \mathrm{TLC}$ versus $\Delta$ Helbich $(\mathrm{R}=0.41, \mathrm{p}=0.02)$. The relationship between $\triangle \mathrm{FEV} 1 / \mathrm{FVC}$ and $\Delta$ Santamaria is shown in figure 1 .

\section{Discussion}

In this study, the ability of five different HRCT-scoring systems and pulmonary function tests were compared to detect and monitor progression of lung damage in 48 children with $\mathrm{CF}$.

The most striking finding of this study is that structural abnormalities as scored on HRCT scans increased significantly, independently of the scoring system used, while lung function parameters remained unchanged or even improved.

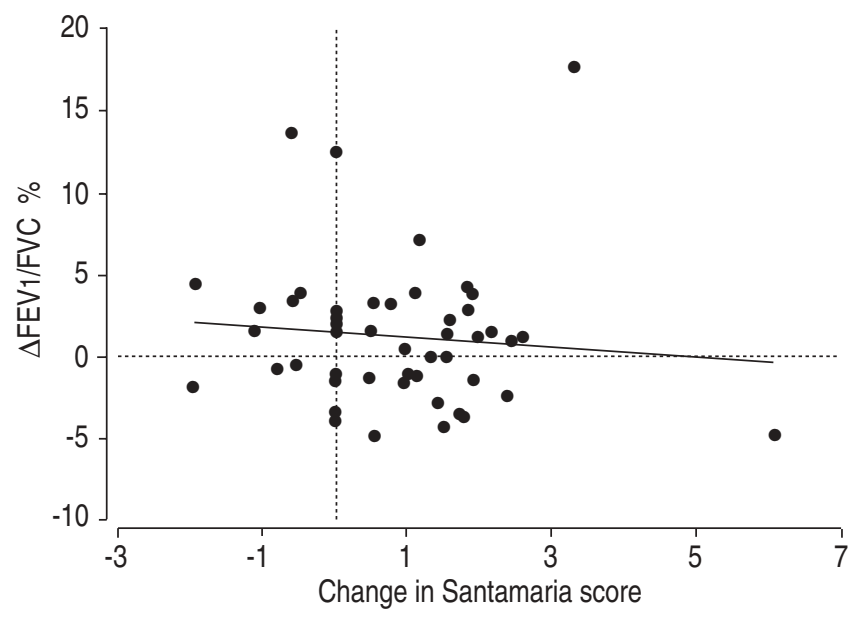

Fig. 1. - The weak correlation $(\mathrm{R}=-0.31, \mathrm{p}=0.04)$ between changes in lung function (forced expiratory volume in one second ( $\mathrm{FEV} 1)$ /forced vital capacity (FVC)) over a 1 -yr period and structural changes on high-resolution computed tomography (HRCT; Santamaria score) is shown. Patients with improved lung function have a change in FEV $1 /$ FVC above 0 , and patients with progressive structural lung damage have a Santamaria score above 0 .
This shows that HRCT was more sensitive than PFT in monitoring $\mathrm{CF}$ lung disease under the assumption that $\mathrm{CF}$ lung disease is progressive. In addition, the authors showed that it was predominantly the severity, extent and peripheral extension of bronchiectasis that worsened significantly. The bronchiectasis most likely represents irreversible structural damage. The irreversibility of bronchiectasis is supported by the fact that none of the patients who had bronchiectasis on HRCT1 were without bronchiectasis on HRCT2.

There are a number of possibilities why the structural abnormalities on HRCT were not reflected by the PFT. Firstly, the signal-to-noise ratio for PFT is likely to be less than that of HRCT since PFT depend more on patient cooperation than HRCT, especially for the younger children, and since PFT are more difficult to perform than a breathhold on the HRCT table. Indeed, the HRCT can be scored even when there is minor motion artefact or some variation in inflation level. Furthermore, the results of PFT are expressed as $\%$ pred with reference to a large population sample and this can introduce variability related to the pubertal growth spurt [11]. In addition, HRCT can detect local abnormalities such as small areas of atelectasis and bronchiectasis that may be functionally insignificant. PFT provide a global estimate of the lung integrity [1]. In this study RV \% pred improved for the whole group, but remained unchanged for the children $>10$ yrs of age. The improvement in RV may, therefore, be a reflection of patient effort. For the young children it is more difficult to reach RV during expiration. However, the RV/ TLC \% and RV/TLC \% pred improved slightly in the children $>10 \mathrm{yrs}$ of age. This suggests that the improvement in RV/ TLC $\%$ pred is only partially caused by an effect of age. The second important finding of this study is that the structural damage was irreversible in most patients irrespective of their change in PFT. In many patients the change in HRCT was dissociated from changes in PFT. Approximately one-half of the patients who had improved lung function had progressive structural lung disease.

The third important finding is that substantial structural lung damage was present on HRCT scans even in children who had lung function within the normal range. This supports the conclusions of earlier studies that conventional PFT are relatively insensitive to detect the onset and early progression of lung disease in CF $[1,7,12]$. These results indicate that the current monitoring strategy using PFT may fail to detect disease progression and suggest that studies 

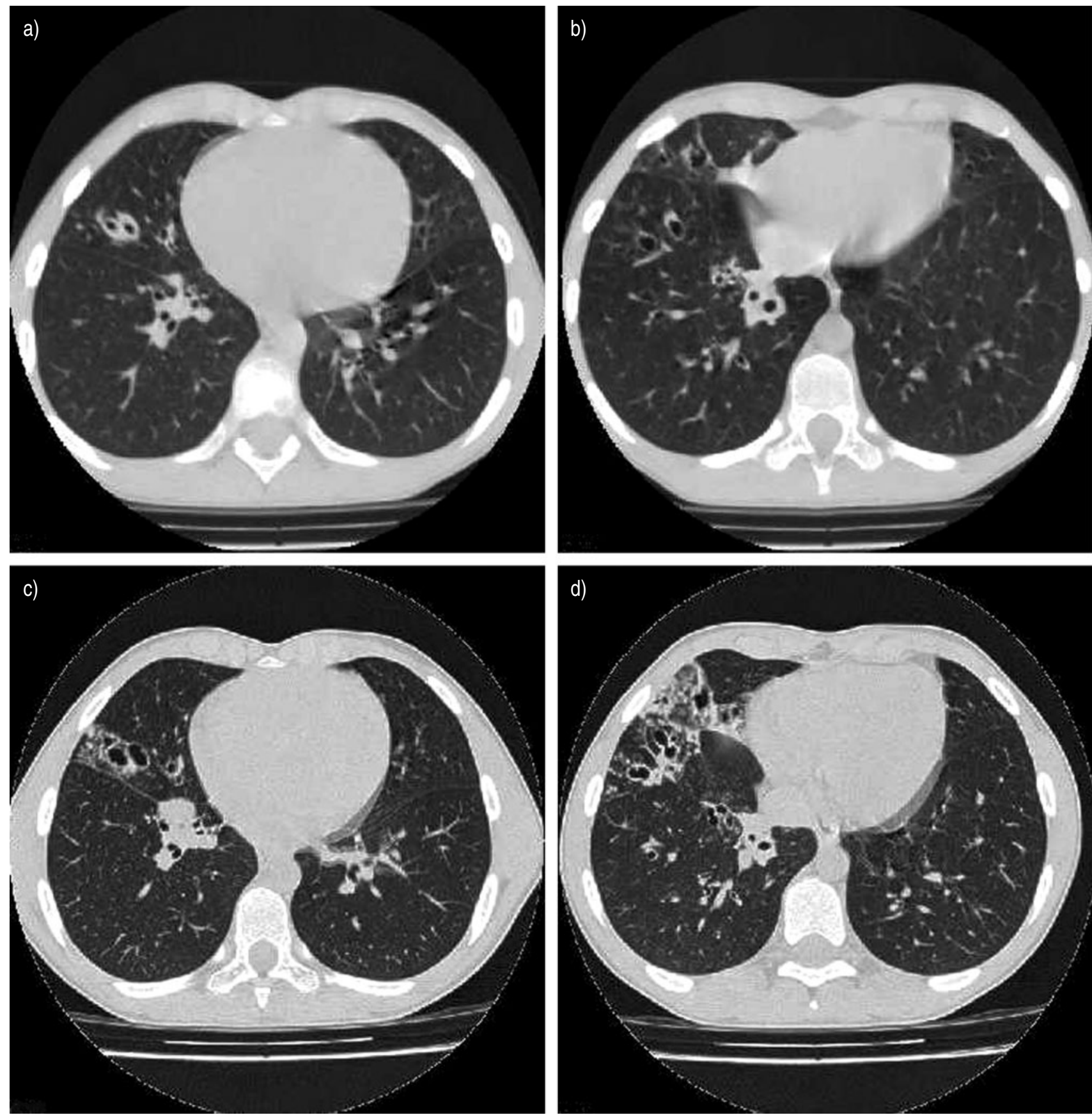

Fig. 2. - Patient with cystic fibrosis with improvement of lung function but detoriation of lung structure. The top panels (a and b) show highresolution computed tomography (HRCT) scan 1 in a patient of 10 yrs of age. Lung function (\% predicted) is as follows: forced expiratory volume in one second $\left(\mathrm{FEV}_{1}\right)$ of 86 , forced vital capacity (FVC) of 93, forced mid-expiratory flow (FEF25-75\%) of 80, and FEV1/FVC of 80. HRCT scores are: Castile 17, Brody 16, Helbich 9, Santamaria 10, and Bhalla 9. The lower panels (c and d) show HRCT2 at 13 yrs of age. Lung function (\% pred) is: FEV1 96, FVC 91, FEF $25-75 \% 105$, and FEV $1 / F V C$ 90. HRCT scores are: Castile 22, Brody 17, Helbich 12, Santamaria 13 , and Bhalla 12

using PFT to monitor treatment response may underestimate treatment effects. Therefore, it is possible that because of flawed outcome measures, the optimal treatment regime to minimise the progression of structural lung damage in $\mathrm{CF}$ patients is as yet unknown.

In this study, the HRCT features that changed most over the 2-yr observation period were the severity score for bronchiectasis and mucous plugging. Selective scoring of these parameters, instead of the full range of parameters included in the currently used complex scoring systems, may be more efficient and reproducible. A simplified scoring system may prove to be adequate for patient management and therapeutic trials. Clearly, such a system should be tested in further studies against the complex scoring systems to test this hypothesis. Monitoring CF patients using HRCT carries potential risk due to the associated radiation exposure. Since the probability of cancer induction from ionising radiation is highest in the paediatric age group [13] and increases with each successive scan, the proposal to employ serial HRCT scans must be balanced against the potential radiation risk. Further research to determine the optimal scan parameters and frequency of HRCT scans for CF monitoring is required. 
This study has some limitations. First, only one observer did the scoring. However, in a previous study, in which this observer participated, it was shown that the within- and between-observer variability for the five scoring systems was good [7]. Secondly, clinical outcomes were not measured in these patients and thus it cannot be assessed whether the worsening on HRCT scans affected the clinical management of the patients. However, a case scenario (fig. 2) illustrates how CT scoring could be used in a clinical setting. In this patient more aggressive therapy may have affected disease progression if it had been detected earlier. Larger prospective clinical studies in which CT scans are used in clinical decision making are required to test the importance of this conjecture.

To the best of the authors' knowledge, this is the first study that shows that HRCT can reveal progressive, irreversible structural damage in the lungs of cystic fibrosis patients over a 2-yr period; changes that are not associated with deterioration in lung function. Since the evolution of lung function over time may be dissociated from changes in the lung structure it may be advantageous to include high-resolution computed tomography in the standard follow-up of cystic fibrosis patients. Furthermore, these data support the use of highresolution computed tomography as a measurement tool in therapeutic trials for computed tomography patients, where the outcome measure is a slowing of progression of lung disease. Conversely, high-resolution computed tomography involves significant radiation exposure and in this era of prolonged patient survival in cystic fibrosis, the risk-benefit ratio for routine high-resolution computed tomography assessment needs to be more fully explored.

Acknowledgements. The authors would like to thank F. Long for reviewing the manuscript.

\section{References}

1. Tiddens HA. Detecting early structural lung damage in cystic fibrosis. Pediatr Pulmonol 2002; 34: 228-231.
2. Castile RG, Hayes JR, Flucke RL, Long FR, McCoy KS Correlation of structural and functional abnormalities in the lungs of infants with cystic fibrosis. Pediatr Pulmonol 2000; 20: A427.

3. Brody AS, Molina PL, Klein JS, Rothman BS, Ramagopal M, Swartz DR. High-resolution computed tomography of the chest in children with cystic fibrosis: support for use as an outcome surrogate. Pediatr Radiol 1999; 29: 731735.

4. Helbich TH, Heinz-Peer G, Eichler I, et al. Cystic fibrosis: CT assessment of lung involvement in children and adults. Radiology 1999; 213: 537-544.

5. Santamaria F, Grillo G, Guidi G, et al. Cystic fibrosis: when should high-resolution computed tomography of the chest be obtained? Pediatrics 1998; 101: 908-913.

6. Bhalla M, Turcios N, Aponte V, et al. Cystic fibrosis: scoring system with thin-section CT. Radiology 1991; 179: 783788.

7. Jong de PA, Ottink MD, Robben SG, et al. Computed tomography assessment of pulmonary disease in children with cystic fibrosis: various scoring system comparisons and bronchial and arterial measurements. Radiology 2004 (in press).

8. Quanjer PH, Borsboom GJ, Brunekreff B, et al. Spirometric reference values for white European children and adolescents: Polgar revisited. Pediatr Pulmonol 1995; 19: 135-142.

9. Wang X, Dockery DW, Wypij D, Fay ME, Ferris BG Jr. Pulmonary function between 6 and 18 years of age. Pediatr Pulmonol 1993; 15: 75-88.

10. Zapletal A, Samanek M, Paul T. Lung Function in Children and Adolescents. Methods, Reference Values. Basel, Karger, 1987.

11. Merkus PJ, Tiddens HA, de Jongste JC. Annual lung function changes in young patients with cronic lung disease. Eur Respir J 2002; 19: 886-891.

12. Maffessanti M, Candusso M, Brizzi F, Piovesana F. Cystic fibrosis in children: HRCT findings and distribution of disease. J Thorac Imaging 1996; 11: 27-38.

13. Brenner DJ, Elliston CD, Hall EJ, Berdon WE. Estimated risks of radiation induced fatal cancer from pediatic CT. AJR Am J Roentgenol 2001; 176: 289-296. 